\title{
基于物联网的智能家居控制系统设计与实现
}

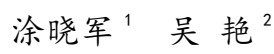 \\ 1 淮安市高级职业技术学校, 江苏淮安 223005 \\ 2 江苏省连水中等专业学校, 江苏淮安 223005
}

[摘要]近年来, 我国社会经济水平得到了显著的提升, 从而使得民众的生活水平得以明显的提升, 这样就为各个领域的发展 壮大创造恶劣良好的基础。物联网其实质就是说将那些具有良好感知能力的物体借助互联网相互连接之后所形成的集合网络。 在科学技术快速发展的推动下, 使得大量的新型科学技术应时而生, 为智能家居行业的良好发展给予了重要的技术支持。借 助互联网将各个物体进行连接, 从而实现信息的共享, 针对人与物进行高度智能化控制是智能家居领域未来发展的主流趋势。 所以, 综合实际情况针对智能家居控制系统的设计展开深入的分析和优化, 从而更好的为人们提供舒适的生活环境。这篇文 章主要围绕基于物联网智能家居控制系统设计展开深入综合分析, 希望能够对我国智能家居领域的发展起到积极的推动作用。 [关键词]物联网; 智能家居; 控制系统; 设计; 实现

DOI：10.33142/sca.v3i6.2490 中图分类号：TP273.5 文献标识码：A

\section{Design and Implementation of Intelligent Home Control System Based on Internet of Things}

\author{
TU Xiaojun ${ }^{1}$, WU Yan ${ }^{2}$
}

1 Huaian Senior Vocational \& Technical School, Huaian, Jiangsu, 223005, China

2 Jiangsu Lianshui Secondary Professional School, Huaian, Jiangsu, 223005, China

\begin{abstract}
In recent years, Chinese social and economic level has been significantly improved, so that the people's living standards can be significantly improved, which creates a bad and good foundation for the development of various fields. The essence of the Internet of Things is to connect those objects with good perception ability through the internet. Driven by the rapid development of science and technology, a large number of new science and technology are born in time, which provides important technical support for the good development of smart home industry. With the help of the internet, various objects are connected to achieve information sharing and highly intelligent control of people and objects is the mainstream trend in the future development of smart home. Therefore, comprehensive actual situation for the design of smart home control system to carry out in-depth analysis and optimization, so as to better provide people with a comfortable living environment. This paper mainly focuses on the design of intelligent home control system based on Internet of things, hoping to play a positive role in promoting the development of smart home in China.
\end{abstract}

Keywords: Internet of Things; smart home; control system; design; implementation

\section{引言}

在社会快速发展的影响下, 使得民众的思想意识出现了明显的变化, 人们对居住环境的需求也在逐渐的提高, 在 这种发展形势下, 智能家居应时而生, 智能家居最为突出的优越性就是便捷性和智能性, 与传统模式的家居相对比来 说具有非常明显的服务性, 并且也是家居领域的发展必然趋势。智能家居控制具有较强的层次性, 涉及到多个分支系 统, 诸如: 中央控制系统、家居终端、小区控制系统, 外层控制系统等等。基于物联网的智能家居控制系统系统是以 提升家居安全性、便利性、舒适性、艺术性, 并实现环保节能的居住环境为目的。以智能化、人性化、高性价比为原 则, 在现有技术上进行创新设计的全新控制系统。借助专业电子设备以及网络对家居设施进行连接, 从而将娱乐、生 活、健康等多项功能融合到智能家居系统之中, 从而有效更好的为人们的生活提供服务。

\section{1 系统总体设计}

具体的设计如下:

\section{1 系统总体方案设计}

就智能家居控制系统来说, 通常都是由三个分支系统组合而成, 首先是机器的分支控制系统, 整个系统涉及到执 行单元、核心处理单元、无线点网络控制器等多种分支配置。其次是网络服务器, 其最为主要的作用就是对服务器终 端的各项信息数据加以控制。再有是移动终端应用程序, 主要包括汶览器终端主机控制终端程序。客户可以借助上位 
机的客户端向着下位计算机管制体系释放指令, 整个系统的运行流程为: 子控制系统内所涉及到的多个无线传感设备 负责对家庭环境信息进行收集, 借助互联网模板将获得的信息传送到服务器。在服务器获取数据之后, 会进行综合整 理。客户利用专门的网络客户终端朝着管制体系释放出指令, 客户端程序借助网络系统向服务器释放指令, 由服务器 按照信息指令来对智能家居系统进行调控。

\section{2 系统硬件架构设计}

整个智能家居控制系统的实时感知无线传感网系统通常都是选择运用的 Zigbee 网络的拓扑结构, 并且借助 igbee 网络节点能够完成对室内温度、光亮、空气质量等重要参数进行收集。整个系统都是由下面几个分支控制模块组合而 成: 首先, 智能灯光模块是由光照传感设备以及节能照明设备组合而成, 主要作用就是针对光照强度实施高效的调控。 其次, 智能家电控制模块, 对整个系统内所设置的电路开关加以控制。再有, 智能温控模块其主要作用就是对室内空 调系统进行适当的调控从而完成对室内温度的管控。还有, 煤气泄漏报警模块, 其作用就是针对室内煤气系统的情况 进行监控。最后, 红外传感器警报模块能够起到防盗的作用, 因为红外线能够被肉眼所看到, 具有良好的安全性和保 密性, 所以可以切实的引用到家居智能系统之中, 能够对人体完成探测。如果所布防的范围内检测到存在人员移动的 情况, 那么这个模块就可以将信息发送到指定的电子终端设备之中。就制定家具控制系统结构来说主要涉及到下面几 个方面: 首先, 核心控制装置利用的是 Arduino 单片机 Atme132u4 芯片, 其拥有储备充足的资源, 能够为系统各个功 能设计工作提供有力的支持。其次, 无线模块其主要作用就是能够实现对智能家居系统进行远距离的操控, 这一模块 在整个智能系统中的作用是非常巨大的。最后, 在系统之中需要设置温度传感器设备, 对系统运行过程中的温度波动 情况进行实时监测。

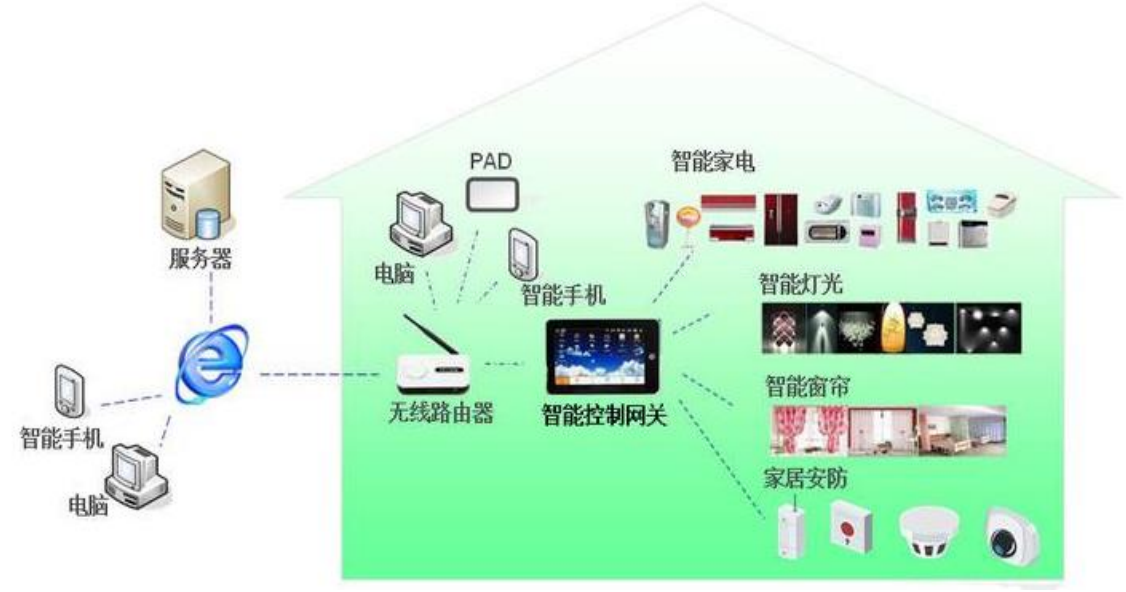

图 1 基于物联网的智能家居控制系统示意图

\section{2 关键技术分析}

\section{1 Android 终端}

在安卓系统之中所设置的内核通常为 Linux, 系统的运行都会以这一内核为中心, 在系统周边会安设专门的底部驱 动设备, 并且会在应用模块中设置专门的应用程序, 将其与整个系统进行连接。应用程序往往都会被人们选择放置在 整个系统的最上层, 通常情况下 Activity 可以呈现出一个单独的界面, 用户可以利用这个界面来完成对应用程序的操 控, 由于整个程序涉及到几个基础功能, 所以每个应用程序都会设计出多个Activity, 并且所有的Activity 会被设计 出不同的功能。 ${ }^{[2]}$

\section{2 Tomcat}

这篇文章中所讲述的智能家居智能系统中选择的是 Tomcat 来充当服务器设备。Tomcat 中融合了专门的微型服务器, 并且其最为突出的特点就是稳定性强, 具备良好的无偿开源。在开启 Tomcat 服务器之后, 启动系统会按照既定的流程 来对各个连接器进行开启, 这个时候连接器都处在等待的状态上。如果客户端在借助浏览器来与服务器进行连接的时 候, 连接器能够将客户所需要的信息传递到模块中, 接收到指令之后, 整个, 整个系统会对数据进行综合分析, 并且 将其传递给适当的主机设备。 


\section{3 Http 协议}

Ht tp 协议最为重要的作用就是将客户端成簇与服务端程度进行连接, 实现二者的通信。这一协议中明确了二者在 进行信息交流的时候需要遵从的数据格式。Http 协议的涉及到的工作如下: 首先, 客户端或者是浏览器与 Web 服务器 完成连接之后, 通常客户端或者是浏览器都会向服务器终端释放指令。其次, 在服务器接收到指令之后, 会对汶览器 给予回应。再有, Web 服务器端在接收到反馈信息之后, 客户端程序或者是汶览器会将接收到的信息依据规定的标准形 式加以展示。

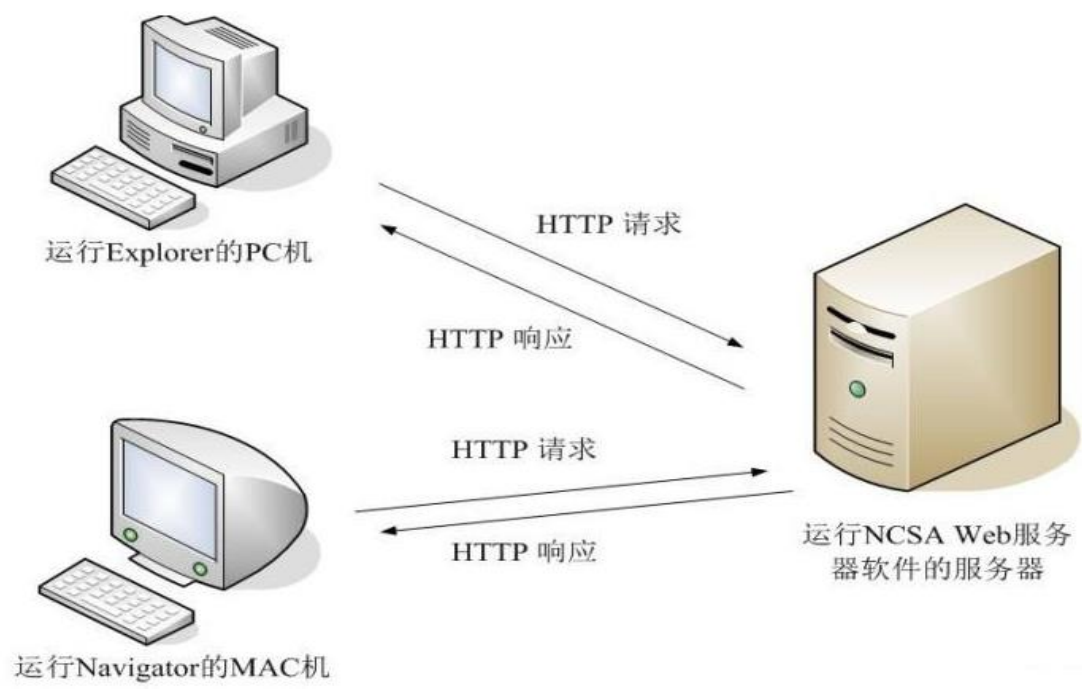

图 2 http 协议响应示意图

\section{4 Web 服务器}

Web 服务器往往都是被安设在整个系统中服务器终端, Web 服务器的主要作用就是向 APP 终端以及浏览器提供需要 的信息。Web 服务器的运行需要人工操作, 客户端与浏览器借助线路与网络进行连接的时候, 服务器会给予辅助, 服务 器不能自行与客户端程序或者是汶览器进行连接。

\section{3 控制系统功能的功能实现}

在实际将控制系统加以使用的时候, 需要利用操作界面来对整个系统进行操控, 这就需要在进行系统前期设计工 作的时候, 针对系统运行可能遇到的各类问题加以模拟, 确保系统可以在遇到各种突发情况的时候都能够有效的加以 解决, 手机端可以及时的对系统进行调控, 从而规避无法处理情况的发生, 控制系统不能自主处理, 并且也无法及时 的上报, 这样就会引发诸多损失情况。上文中针对控制系统的各个团建和硬件安设提出了设计思路, 在物联网的影响 下, 综合软件硬件的辅助, 能够将系统的各项功能充分的加以利用。诸如: 客户想要对家居数据进行利用和查阅, 可 以自行登录系统网址, 输入权限并综合智能家电传感器对智能家居终端进行验证, 要想保证上述操作有序的开展, 那 么需要确保多个层次之间的稳定性，确保开关控制，及时得到各终端反馈。

\section{4 结束语}

科学技术水平的不断提升, 使得智能家居客户对家居设计突出了更高的要求, 这样也使得纯铜家居设计中所存在 的各种问题越发的凸现出来。智能控制设计能够有效的促进家居设计效果的提升, 所以设计工作人员务必要充分结合 实际情况和需要提升家居控制系统的运行效率，更好的满足人们对智能家居的需要。

\section{[参考文献]}

[1] 翟冠杰. 基于物联网技术的智能家居系统设计与实现 $[\mathrm{J}]$. 电子技术与软件工程, 2018(23): 2 .

[2]荀艳丽,焦库, 张秦菲. 基于物联网的智能家居控制系统设计与实现 [J]. 现代电子技术, 2018,41(10): 74-76.

$[3]$ 左翰博. 基于物联网的智能家居控制系统设计与实现 $[\mathrm{J}]$. 电脑迷, 2018(01): 76-77.

[4]杨堤.基于物联网的智能家居控制系统设计与实现 [J].电子世界,2012 (21) : 16-17.

作者简介: 涂晓军 (1982-), 男, 中国矿业大学, 研究生, 通信与信息系统专业, 现任淮安市高级职业技术学校教师, 8 年, 讲师。 